\title{
Design and Synthesis of Quasi Dual-mode, Elliptic Coaxial Filter
}

\author{
Sovuthy CHEAB, Peng Wen WONG
}

Dept. of Electrical and Electronic Engineering, University of Technology Petronas, 326100, Tronoh, Perak, Malaysia

sovuthy@gmail.com,wong_pengwen@petronas.com.my

\begin{abstract}
This article introduces the design of a novel quasi dual-mode, elliptic coaxial filter. The transfer function is mapped to a Generalized Chebyshev prototype with symmetrically located transmission zeros (TZs) where the coupling values are extracted. Furthermore, the miniaturization is achieved by incorporating stepped-impedance coaxial line with inductive element shunted at the center to exhibit a quasi dual-mode property. Theoretical analysis together with experimental prototype is presented. The center frequency of the filter is $2.7 \mathrm{GHz}$. The simulated and measured insertion loss/return loss are $1.2 \mathrm{~dB} / 15 \mathrm{~dB}$ and $2.5 \mathrm{~dB}$ $111.5 \mathrm{~dB}$ respectively. Both theoretical and measured results show a very good agreement.
\end{abstract}

\section{Keywords}

Bandpass filter, coaxial filter, elliptic, quasi dual-mode, source-load coupling

\section{Introduction}

Microwave filter with compact size, low weight, low loss, and high power handling and selectivity is demanded specifically in cellular communications base-stations. With these stringent requirements, the planar based microstrip filter [1], [2], [3], [4] cannot be used due to its inherent low $Q$-factor. The most suitable transmission line for this application is coaxial transmission line for its capability of larger usable bandwidth, smaller size and high $Q$-factor with TEM wave propagation. However, to date the conventional coaxial filter developed could achieve only one resonance per physical structure [5], [6]. This single mode technology based on combline filters are widely employed in base station [7]. The major disadvantage associated with the conventional single mode technology is the relatively large filter size and high material cost. Until recently the attempt to realize two resonances and dual bands response using coaxial had been reported based on combline topology [8]. The structure is made up of three metallic conductors: the inner and the intermediate conductors and the enclosure. The two resonances achieved have to be dual bands due to the limitation of the coupling of the resonator structure.
In terms of selectivity, elliptic and pseudo-elliptic filters offer optimal solutions to filtering function with high selectivity and low in-band insertion loss. This is achieved by shifting the TZs of an N-degree filter network from infinite frequencies to finite frequencies. This article presents the design of quasi dual-mode, elliptic coaxial bandpass filter. As miniaturization is one of the key requirements for a filter, a novel stepped impedance dual mode resonator is adopted where a compact design is shown. A prototype is realized and presented in this paper to demonstrate the feasibility of the approach. It will be shown that it is possible to have two resonances for one physical resonator structure for coaxial TEM line. Moreover, the source-load coupling gives additional two transmission zeros (TZs) beyond the passband which offers a quasi-elliptic response.

The organization of this paper is as follows: Section 2 describes a brief theory of quasi-dual mode, elliptic filter including the mapping of the transfer function to the Generalized Chebyshev prototype. Section 3 discusses the coaxial prototype design together with result analysis. Finally, Section 4 is the conclusion.

\section{Theory}

Depicted in Fig. 1 is the coupling and routing structure of dual mode resonator with source-load coupling where $K_{1}$, $K_{11}$, and $K_{S L}$ are the inverters.

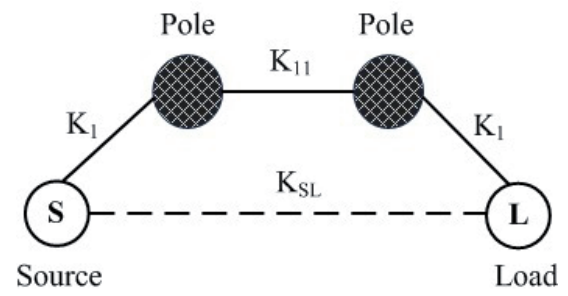

Fig. 1. Coupling and routing structure.

The low-pass section comprises of two capacitors $C_{1}$ and $C_{2}$ with an inverter $K_{11}$ in between. The two resonators are coupled to the source or load by an inverter $K_{1} . K_{S L}$ is introduced to form the source-load coupling. The circuit model of the upper path of Fig.1 is shown in Fig.2. 


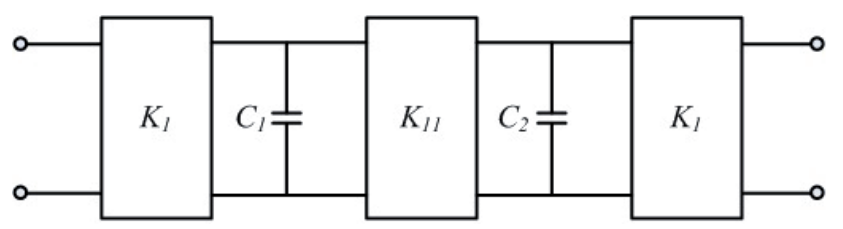

Fig. 2. Circuit model of the low-pass dual mode resonator (without source-load coupling).

Assuming that the capacitor $C_{1}=C_{2}=1 \mathrm{~F}$, the cascading elements in Fig. 2 builds up the transfer matrix as

$$
\mathbf{T}_{1}=\left[\begin{array}{cc}
\omega K_{11} & j\left(-\frac{K_{l}^{2}}{K_{11}}+K_{l}^{2} \omega^{2} K_{11}\right) \\
-j \frac{K_{11}}{K_{l}^{2}} & \omega K_{11}
\end{array}\right] .
$$

Note that $j$ is used in this paper to denote the imaginary unit of a complex number which is the square root of -1 . The corresponding $\mathbf{Y}$ matrix is

$$
\mathbf{Y}_{1}=\left[\begin{array}{cc}
\frac{-j \omega K_{l 1}^{2}}{K_{l}^{2}\left(-1+\omega^{2} K_{l 1}^{2}\right)} & \frac{j K_{l 1}}{K_{l}^{2}\left(-1+\omega^{2} K_{l 1}^{2}\right)} \\
\frac{j K_{l 1}}{K_{l}^{2}\left(-1+\omega^{2} K_{l 1}^{2}\right)} & \frac{-j \omega K_{l 1}^{2}}{K_{l}^{2}\left(-1+\omega^{2} K_{l 1}^{2}\right)}
\end{array}\right]
$$

The transfer matrix of the lower path of Fig. 1 (source-load coupling) is

$$
\mathbf{T}_{S L}=\left[\begin{array}{cc}
0 & j K_{S L} \\
\frac{j}{K_{S L}} & 0
\end{array}\right] .
$$

The corresponding $\mathbf{Y}$ matrix is

$$
\mathbf{Y}_{S L}=\left[\begin{array}{cc}
0 & \frac{j}{K_{S L}} \\
\frac{j}{K_{S L}} & 0
\end{array}\right]
$$

The total $\mathbf{Y}$ matrix is

$$
\mathbf{Y}_{T}=\mathbf{Y}_{1}+\mathbf{Y}_{S L}
$$

The conversion between two-port network parameters table in [9] is used to convert from $\mathbf{Y}$ to $\mathbf{S}$-parameter. Finally, the transfer function of the network is

$$
\left|S_{12}(j \omega)\right|^{2}=4\left|\frac{\gamma_{2} \omega^{2}+\gamma_{0}}{\rho_{2} \omega^{2}+\rho_{1} \omega+\rho_{0}}\right|^{2}
$$

where

$$
\begin{gathered}
\gamma_{0}=K_{1}^{2}\left(K_{11} K_{S L}-K_{1}^{2}\right) K_{S L} \\
\gamma_{2}=K_{1}^{4} K_{11}^{2} K_{S L}
\end{gathered}
$$

$$
\rho_{0}=-2 K_{11} K_{S L} K_{1}^{2}+K_{S L}{ }^{2} K_{1}^{4}+K_{1}{ }^{4}+K_{11}{ }^{2} K_{S L}{ }^{2},
$$

$$
\begin{gathered}
\rho_{1}=2 j K_{S L}{ }^{2} K_{1}{ }^{2} K_{11}{ }^{2}, \\
\rho_{2}=-K_{1}{ }^{4} K_{11}{ }^{2}-K_{S L}{ }^{2} K_{1}{ }^{4} K_{11}{ }^{2} .
\end{gathered}
$$

Now consider the transfer function for a lossless passive filter network which is defined as

$$
\left|S_{12}(j \omega)\right|^{2}=\frac{1}{1+\varepsilon^{2} F_{N}^{2}(\omega)}
$$

where $\varepsilon$ is a ripple constant and $F_{N}(\omega)$ represents a filtering or characteristic function. The expression of $F_{N}(\omega)$ is given by [10]

$$
F_{N}(\omega)=\frac{1}{2}\left[\prod_{r=1}^{N}\left(\alpha_{n}+\beta_{n}\right)+\prod_{r=1}^{N}\left(\alpha_{n}-\beta_{n}\right)\right]
$$

where

$$
\begin{gathered}
\alpha_{n}=\left(\omega-\frac{1}{\omega_{n}}\right)\left(1-\frac{\omega}{\omega_{n}}\right)^{-1} \\
\beta_{n}=\sqrt{\left(\omega^{2}-1\right)\left(1-\frac{1}{\omega_{n}^{2}}\right)}\left(1-\frac{\omega}{\omega_{n}}\right)^{-1} .
\end{gathered}
$$

$N$ is the degree of filter, and $\omega_{n}$ is the position of the $n$-th $\mathrm{TZ}$ in the complex frequency plane. Therefore, the filtering function of the second-order Generalized Chebyshev filter prototype with $15 \mathrm{~dB}$ return loss level and two symmetrically placed TZs at $\pm j 5$ is

$$
F_{N}(\omega)=\frac{49 \omega^{2}-25}{-\omega^{2}+25} .
$$

The filtering function of the quasi dual-mode, elliptic filter is derived from (6) as

$$
f_{N}(\omega)=k\left(\frac{a_{2} \omega^{2}+a_{0}}{b_{2} \omega^{2}+b_{0}}\right)
$$

where $k=1.2$ is the normalizing constant and

$$
\begin{gathered}
a_{0}=2.7 K_{1}{ }^{4}+K_{11}{ }^{2} K_{S L}{ }^{2}-2 K_{11} K_{S L} K_{1}{ }^{2}-K_{S L}{ }^{2} K_{1}{ }^{4}, \\
a_{2}=2.7 K_{1}{ }^{4} K_{11}{ }^{2}\left(K_{S L}{ }^{2}-1\right), \\
b_{0}=\left(K_{11} K_{S L}-1.0 K_{1}{ }^{2}\right) K_{S L} K_{1}{ }^{2}, \\
b_{2}=K_{1}{ }^{4} K_{11}{ }^{2} K_{S L} .
\end{gathered}
$$

It should be noticed that the source-load coupling is introduced so that the filtering function can form a fraction which enables the generation of the finite-frequency TZs at both sides of the passband. The mapping gives $K_{1}=0.831$, $K_{11}=0.552$, and $K_{S L}=-8.276$. The transfer function of the quasi-elliptic dual-mode network expressed in (6) then becomes 


$$
\left|S_{12}(j \omega)\right|^{2}=\frac{0.012754 \omega^{4}-0.63771 \omega^{2}+7.9714}{\omega^{4}-1.6372 \omega^{2}+8.1280},
$$

and its corresponding reflection function is

$$
\left|S_{11}(j \omega)\right|^{2}=\frac{\omega^{4}-1.020408163 \omega^{2}+0.2603082049}{\omega^{4}-1.637235378 \omega^{2}+8.128002235} .
$$

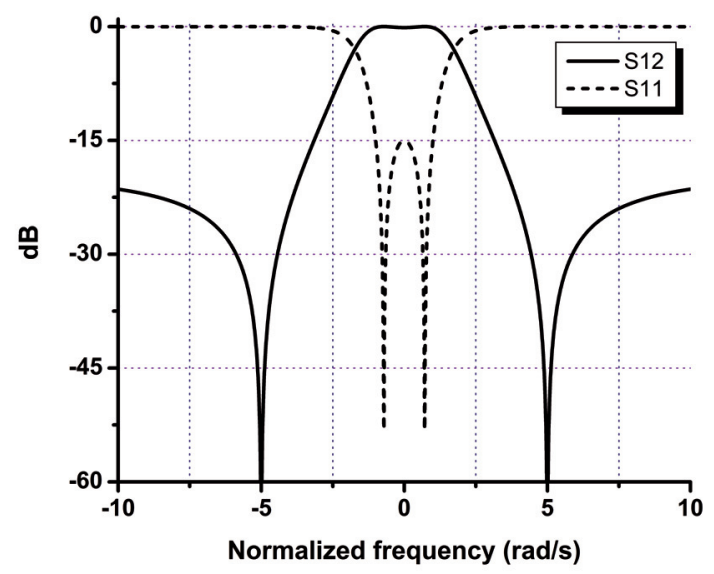

Fig. 3. Normalized frequency responses.

The normalized frequency responses in $\mathrm{dB}$ of the transfer and reflection function for two paths is illustrated in Fig. 3 where the passband return loss is $15 \mathrm{~dB}$ and the two poles within the passband and two TZs in the stopband are clearly shown.

\section{Coaxial Prototype}

The quasi dual-mode, elliptic TEM coaxial bandpass filter prototype has been fabricated using solid aluminum filled by the air as the dielectric. Fig. 4 depicts the structure layout of the filter prior to fabrication using $\mathrm{CNC}$ machine whereas Fig. 5 gives the physical dimensions of the coaxial filter prototype after correcting the capacitive coupling between the input/output to the resonator.

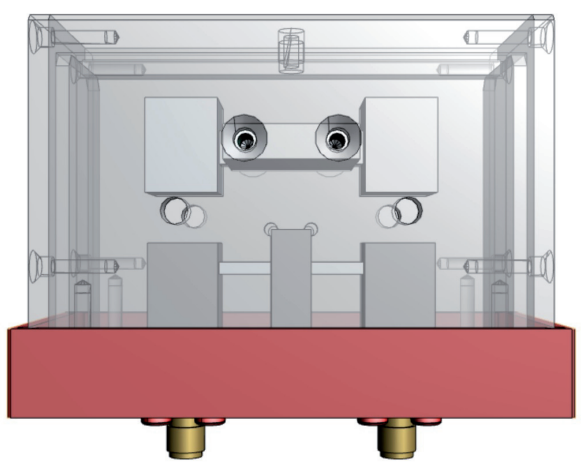

Fig. 4. The structure layout of quasi dual-mode, elliptic coaxial filter.

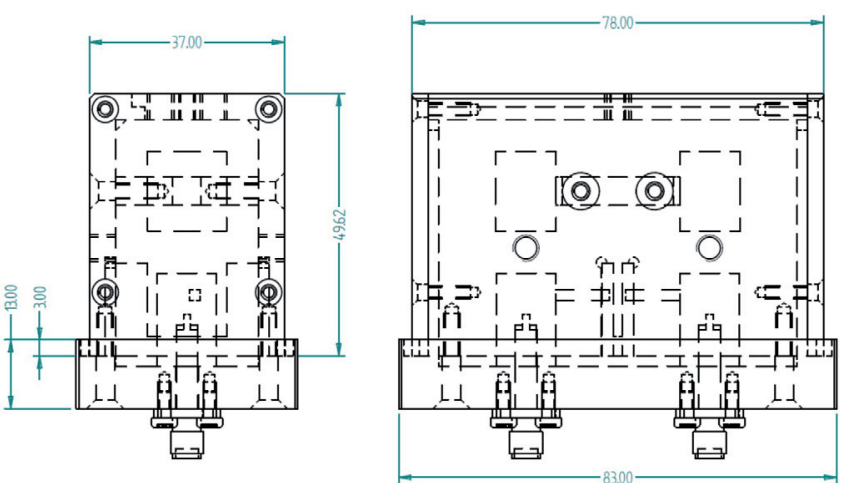

Fig. 5. Physical dimensions. (Unit: millimeters).

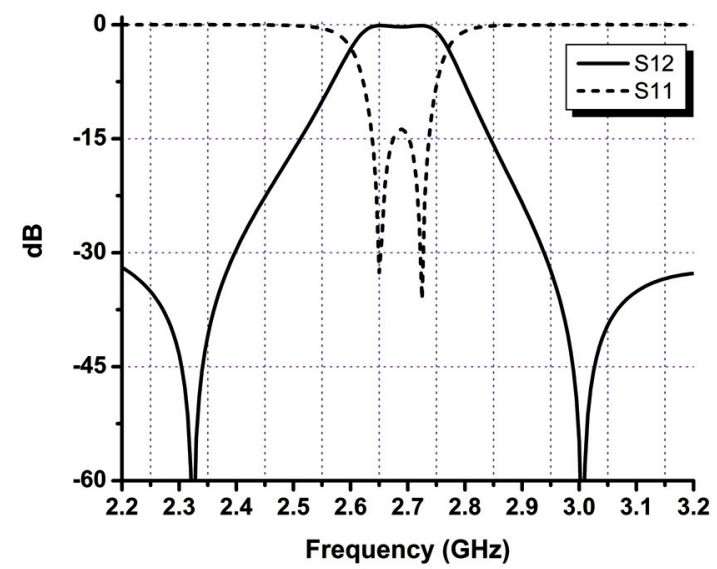

Fig. 6. Simulation transmission and reflection responses.

Figure 6 shows the simulated response of quasi dual-mode, elliptic coaxial filter using ANSYS HFSS [11]. The fabricated coaxial bandpass filter prototype is shown in Fig. 7. The bandpass filter is smaller in size due to the use of the stepped-impedance but with the expense of its $Q$-factor reduction. Based on the graph in [12], the $Q$-factor of the stepped impedance resonator is reduced by $15 \%$ if the impedance ratio is 1.3 . Hence the resulting $Q$-factor is only 2450.

The measured transmission and reflection response for the filter is shown in Fig. 8. It was successfully demonstrated that the filter gives two poles centered at $2.7 \mathrm{GHz}$ with $4 \%$ passband bandwidth and two TZs beyond the passband. The measured minimum insertion loss is $2.5 \mathrm{~dB}$ and the return loss is better than $11.5 \mathrm{~dB}$ which is higher as compare to the simulated response with the insertion loss of $1.2 \mathrm{~dB}$ and return loss of $15 \mathrm{~dB}$. This higher insertion loss is mainly because the each $\mathrm{TZ}$ at both sides beyond the passband from the measurement are very much closer to the passband compared to those obtained from simulations. The presence of 


\begin{tabular}{|c|c|c|c|c|c|}
\hline Ref. & Resonator structure & No. of resonances & $Q$ & Selectivity & TZ \\
\hline$[13]$ & Combline (coax) & 1 (per unit structure) & High & High & No \\
\hline$[14]$ & Combline (coax) & 1 (per unit structure) & High & High & Yes \\
\hline$[15]$ & Dual-mode (coax) & 2 (per unit structure) & High & High & No \\
\hline$[16]$ & Dual-mode (planar microstrip) & 2 (per unit structure) & Low & Moderate & Yes \\
\hline This work & Quasi dual-mode, elliptic (coax) & 2 (per unit structure) & High & High & Yes \\
\hline
\end{tabular}

Tab. 1. Comparison of state-of-the-art.

the stub to achieve the source-load coupling gives rise of two finite-frequency TZs on both sides of the passband as presented. The location of these TZs can be easily controlled by changing the dimension of this stub used for the source load coupling.

The comparative study of the main features of the proposed quasi dual-mode, elliptic coaxial filter with the stateof-the-art is given in Tab. 1.
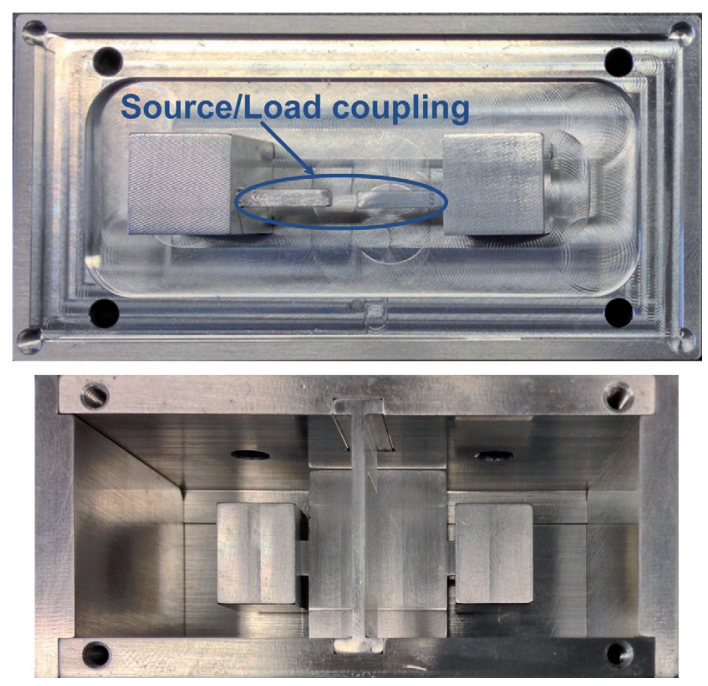

Fig. 7. Quasi dual-mode, elliptic coaxial filter prototype.

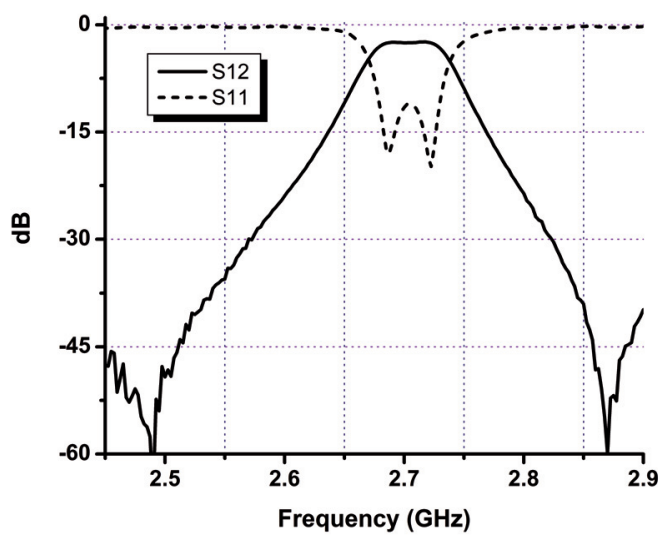

Fig. 8. Measured transmission and reflection responses.

\section{Conclusion}

In this article, a novel technique to construct a quasi dual-mode coaxial bandpass filter is introduced. The miniaturization is obtained by incorporating stepped-impedance coaxial line with inductive element shunted at the center to exhibit a quasi-dual mode property. The experimental prototype is fabricated and shown. The measured results show the two resonances in the passband. It was successfully demonstrated that the filter can achieve a quasi dual-mode, elliptic response with two transmission zeros and two transmission poles. The experimental work shows a good agreement with the theory.

\section{References}

[1] HONG, J. S., LANCASTER, M. J. Microstrip Filters for RF/Microwave Applications. John Wiley \& Sons, Inc. 2001.

[2] BARAL, R. N., SINGHAL, P. K. Recent techniques in design and implementation of microwave planar filters. Radioengineering, 2008, vol. 17 , no. 4 , p. 392-396.

[3] VAGNER, P., KASAL, M. A novel bandpass filter using a combination of open-loop defected ground structure and half-wavelength microstrip resonators. Radioengineering, 2010, vol. 19, no. 3, p. 392396.

[4] DURAN-SINDREU, M., VELEZ, P., BONACHE, J., MARTIN, F. Broadband microwave filters based on open split ring resonators (OSRRs) and open complementary split ring resonators (OCSRRs): Improved models and design optimization. Radioengineering, 2011, vol. 20 , no. 4 , p. $775-784$.

[5] MATthaEI, G. L., YOUNG, L., JONES, E. M. T. Microwave Filters, Impedance-Matching Networks, and Coupling Structures. Norwood (MA): Artech House, 1980.

[6] MATTHAEI, G. L. Comb-line bandpass filters for narrow or moderate bandwidth. Microwave Journal, 1963, vol. 6, p. 82-91.

[7] MANSOUR, R. R. Filter technologies for wireless base stations. IEEE Microwave Magazine, 2004, vol. 5, no. 1, p. 68-74. DOI: 10.1109/MMW.2004.1284945.

[8] RUIZ-CRUZ, J. A., FAHMI, M. M., MANSOUR, R. R. Dualresonance combline resonator for dual-band filters. In IEEE MTT$S$ International Microwave Symposium Digest (MTT). Montreal (Canada), 2012 , p. 1-3. DOI: 10.1109/MWSYM.2012.6259436

[9] POZAR, D. M. Microwave Engineering. John Wiley, 2000, p. 187.

[10] CAMERON, R. J., KUDSIA, C. M., MANSOUR, R. R. Microwave Filters for Communication Systems: Fundamentals, Design and Applications. New Jersey: John Wiley \& Sons, 2007.

[11] HFSS, 3D Full Wave Electromagnetic Field Simulation, Ansoft Designer (R). 
[12] MAKIMOTO, M., SADAHIKO, Y. Corrections to compact bandpass filters using stepped impedance resonators. Proceeding of the IEEE, 1979, vol. 67, no. 11, p. 1568, DOI: 10.1109/PROC.1979.11521

[13] SH-ASANJAN, D., MANSOUR, R. R. A novel coaxial resonator for high power applications. In $44^{\text {th }}$ European IEEE MTT-S International Microwave Conference (EuMC). Rome (Italy), 2014, p. 295 298. DOI: 10.1109/EuMC.2014.6986428

[14] WOLANSKY, D., TKADLEC, R. Coaxial filters optimization using tuning space mapping in CST Studio. Radioengineering, 2011, vol. 20, no. 1, p. 289-294.

[15] CHEAB SOVUTHY, WONG, P. W. Stepped impedance dual mode coaxial filter. In IEEE International $R F$ and Microwave Conference (RFM). Penang (China), 2013, p. 165-167. DOI: 10.1109/RFM.2013.6757240

[16] ATHUKORALA, L., BUDIMIR, D. Compact dual-mode open loop microstrip resonators and filters. IEEE Microwave and Wireless Components Letters, 2009, vol. 19, no. 11, p. 698-700. DOI: 10.1109/LMWC.2009.2032003

\section{About the Authors...}

Sovuthy CHEAB was born in Battambang, Cambodia, in 1986. He received his B.Eng. (Honors) degree and M.Sc degree in Electrical and Electronics both from Universiti Teknologi PETRONAS, Malaysia respectively in 2011 and 2012. He is currently working towards the Ph.D. degree in Microwave Engineering at Universiti Teknologi PETRONAS, Malaysia. His research interests include includes passive microwave filters in planar and cavity design.

Peng Wen WONG was born in Perak, Malaysia, in 1984. He received the B.Eng. (Hons. First-Class) degree from University of Leads in 2005 in Electrical and Electronic Engineering. He then joined Intel Malaysia and worked as a Test R\&D engineer. He received research funding from Ministry of Defense, UK where he completed his Ph.D. in University of Leads in 2009. He is currently a senior lecturer in Universiti Teknologi PETRONAS at the department of Electrical and Electronic Engineering, , teaching electromagnetism and microwave engineering subjects. His research interest includes passive and active microwave filters. He also investigates the applications of microwaves in biology. 